\title{
Caracterización de la hipotonía-hiporrespuesta post vacuna pentavalente y tamizaje del neurodesarrollo en niños bajo 6 años de edad, 2013-2018, Uruguay
}

\author{
Characterization of hypotonia-hyporesponsiveness after pentavalent vaccine and \\ neurodevelopmental screening in children under 6 years of age, 2013-2018, Uruguay
}

\author{
Carlos Zunino', Noelia Speranza2,6, Helena Deutsch³, Ricardo Mora ${ }^{3}$, Cecilia Rebellato3, \\ Gabriela Cuello4, Maite Inthamoussu5, Gustavo Giachetto y Teresa Picón ${ }^{6}$
}

'Departamento de Pediatría. Facultad de Medicina. Universidad de la República.

${ }^{2}$ Departamento Farmacología y Terapéutica. Facultad de Medicina. Universidad de la República.

${ }^{3}$ Ex Residente de Pediatría. Facultad de Medicina. Universidad de la República.

${ }^{4}$ Residente de Epidemiología. Facultad de Medicina. Universidad de la República.

${ }^{5}$ Departamento Farmacología y Terapéutica. Facultad de Medicina. Universidad de la República.

${ }^{6}$ Programa Nacional de Vacunaciones. Ministerio de Salud Pública.

Conflictos de interés: no hubo.

Financiamiento: no hubo.

Recibido: 16 de julio de 2020 (segunda versión: 31 de enero de 2021) / Aceptado: 23 de febrero de 2021

\section{Resumen}

Introducción: La hipotonía-hiporrespuesta (HHR) es uno de los efectos adversos supuestamente atribuibles a la vacunación e inmunización de tipo neurológico más notificados. El impacto a largo plazo a nivel del neurodesarrollo no es completamente conocida. Objetivo: Caracterizar los eventos de HHR post vacuna pentavalente notificados entre 2014 y 2018 al Ministerio de Salud Pública (MSP) de Uruguay. Realizar el tamizaje del neurodesarrollo de los que al momento de la evaluación tenían menos de 6 años de edad. Metodología: Estudio descriptivo de las notificaciones al Sistema Nacional de Farmacovigilancia del MSP. Se realizó el tamizaje del neurodesarrollo con la Guía Nacional para la Vigilancia del Desarrollo. Resultados: 30 casos, la mayoría de breve duración, en las primeras horas post primera dosis y con recuperación espontánea. Requirieron hospitalización 29. Se realizó el tamizaje del neurodesarrollo en 16. La media de tiempo entre el evento y esta evaluación fue 2 años y 2 meses. Fue normal la prueba de tamizaje en 15. En uno se detectó un retraso del lenguaje. Conclusiones: Los episodios de HHR se presentaron con características similares a las descritas en la bibliografía. A pesar de las limitaciones del estudio, no se encontraron retrasos ni desvíos del desarrollo en los niños evaluados.

Palabras clave: hipotonía-hiporrespuesta; vacuna antipertussis; farmacovigilancia.

\section{Abstract}

Background: Hypotonic-hyporesponsive episodes (HHE) is one frequently reported neurologic adverse effect supposedly attributable to vaccination and immunization. Its long-term impact on neurodevelopment is not completely known. Aim: To characterize the post-pentavalent vaccine HHE events reported to the Uruguayan Ministry of Health (M of H) between 2014 and 2018. To perform neurodevelopment screening of those who were under 6 years of age at the time of evaluation. Methods: Descriptive study of the reports made to the National Farmacosurveillance System of the M of $\mathrm{H}$. Neurodevelopment screening was performed using the National Guidelines for Developmental Surveillance. Results: 30 cases were studied. Most cases occurred after the first doses, were of short duration and during the first hours after vaccination, with spontaneous recovery. Median time between the event and this evaluation was 2 years and 2 months. Screening tests were normal in 15. Delay in the language area was detected in one case. Conclusions: HHE events had similar characteristics to those described in the literature, with no severe short-term complications. Despite the limitations of the present study, no delays nor deviations were found in the development of the children who were evaluated.

Keywords: hypotonic-hyporesponsive episodes; antipertussis vaccine; farmacosurveillance. 
tración de la primera dosis de la vacuna dentro de las primeras $24 \mathrm{~h}$ y dura entre 6 y $30 \mathrm{~min}^{6-9,11}$.

Este ESAVI no constituye una contraindicación absoluta sino una precaución para recibir futuras dosis del componente pertussis de la vacuna $\mathrm{DPT}^{12,13}$. Si bien el principal riesgo luego de su ocurrencia es la reiteración, el impacto sobre la salud de los niños, principalmente a nivel del neurodesarrollo, no se conoce completamente. No se disponen de estudios recientes que evalúen a largo plazo el neurodesarrollo de los niños que presentaron HHR. Estos aspectos provocan gran preocupación y ansiedad en las familias ${ }^{11}$.

Es pertinente complementar la información aportada por el Sistema Nacional de Farmacovigilancia mediante la realización de un seguimiento de los eventos a largo plazo que incluya la evaluación del neurodesarrollo. Esta información podría contribuir a la evidencia sobre la seguridad de la vacuna pentavalente.

\section{Objetivos}

- Caracterizar los eventos de HHR post vacuna pentavalente notificados entre 2014 y 2018 al Sistema Nacional de Farmacovigilancia del MSP de Uruguay.

- Realizar el tamizaje del neurodesarrollo de aquellos niños que al momento de la evaluación tenían menos de 6 años. pasiva de ESAVI, enmarcado en el Sistema Nacional de Farmacovigilancia. En promedio se reciben 78 notificaciones por año. Uno de los ESAVI más notificados corresponde a hipotonía-hiporrespuesta (HHR), relacionados comúnmente con la vacuna pentavalente, que incluye las vacunas DPT- HB- Hib (difteria, tosferina, tétanos, hepatitis B, Haemophilus influenzae tipo b) administrada a los 2, 4, 6 y 15 meses de vida, registrándose un aumento en la incidencia de notificaciones en $2018^{4,5}$.

El evento de HHR se caracteriza por la instalación brusca de hipotonía generalizada acompañada de menor respuesta a estímulos sensoriales y cambios en la coloración de la piel, palidez o cianosis. Si bien se lo ha asociado con distintas vacunas (contra $H$. influenzae tipo $\mathrm{b}$, difteria, tétanos, poliomielitis, hepatitis B), se observa más frecuentemente luego de la administración de la vacuna contra la tosferina, principalmente con la vacuna celular $^{6-9}$. La fisiopatología del evento no se conoce aún. Se estima que sea multifactorial, si bien es posible que existan factores de riesgo, tanto de los componentes de la vacuna como intrínsecos del niño $0^{9-11}$.

El diagnóstico de HHR es clínico y se basa en la presentación de la tríada antes mencionada, en un niño que ha recibido recientemente alguna de las vacunas potencialmente implicadas ${ }^{8}$. Según la Brighton Collaboration, los probables eventos de HHR en niños bajo 2 años de edad se pueden clasificar en tres niveles de certeza según sus características clínicas 9 .

Según los reportes de casos publicados, la HHR suele presentarse más frecuentemente luego de la adminis-

\section{Metodología}

Para la caracterización de los eventos HHR post vacuna pentavalente entre los años 2014 y 2018 se captaron a través del Sistema Nacional de Farmacovigilancia del MSP todos los niños que tenían dos años o menos al momento de la notificación. Se incluyeron los que al momento de realizar el tamizaje del neurodesarrollo tenían menos de 6 años.

Se utilizó como definición operativa de HHR y la clasificación en niveles de certeza de la Brighton Collaboration ${ }^{9}$ (Tabla 1).

Las variables para caracterizar los eventos fueron: edad, sexo, características clínicas (cianosis, palidez, cambios del tono, hipo-reactividad, fiebre, movimientos anormales u otros síntomas acompañantes), latencia entre la vacunación y la aparición de éste, duración del episodio, necesidad y tipo de tratamiento, hospitalización, solicitud de exámenes de laboratorio y reiteración en el mismo episodio y recurrencia con dosis posteriores de la vacuna. Se recabó además la finalización del esquema vacunal según edad actual. Se utilizaron como fuentes de datos las notificaciones del Sistema Nacional de Farmacovigilancia y el Sistema Informático de Vacunas del MSP.

Para el tamizaje del neurodesarrollo se utilizó la Guía Nacional para la Vigilancia del Desarrollo del Niño y 


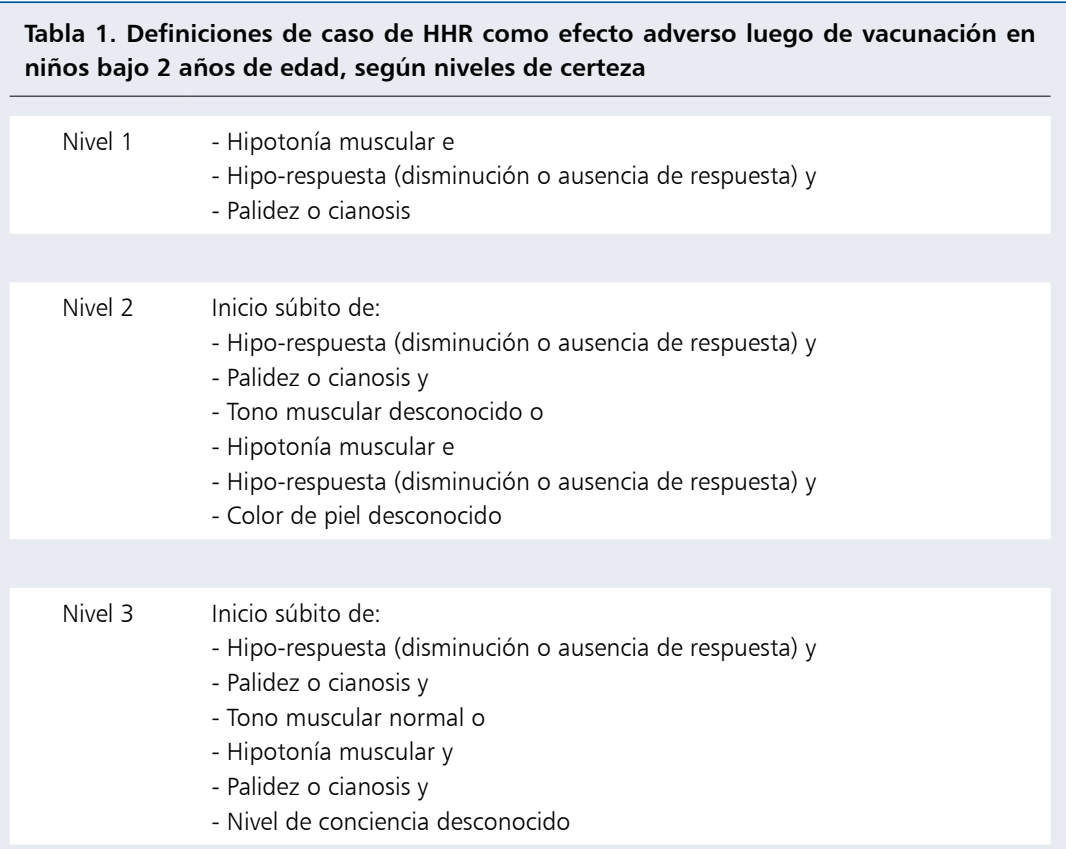

Fuente: The Brighton Collaboration HHE Working Group. "Hypotonic-hyporesponsive episode (HHE) as an adverse event following immunization in early childhood: Case definition and guidelines for data collection, analysis, and presentation". de la Niña Menores de 5 Años, del Ministerio de Salud Pública, publicada en el año 2019 previa validación ${ }^{14}$. Su aplicación fue realizada por tres de los autores, estudiantes del postgrado de pediatría, previamente entrenados, con el niño en buen estado de salud. Se coordinó telefónicamente la evaluación en los domicilios durante el año 2019.

\section{Análisis estadístico}

Se realizó estadística descriptiva para las variables de estudio. Se aplicó la prueba contraste para proporciones para comparar los niños en los que se pudo evaluar el neurodesarrollo con los que no fue posible, las medianas de duración de los eventos entre ambos grupos se compararon utilizando la prueba de U de Mann-Whitney. Se consideró estadísticamente significativo $\mathrm{p}<0,05$. Para el análisis estadístico fueron Excel 2016 y Stata/IC versión 15.

Se solicitó consentimiento informado a la madre, padre o tutor del niño para utilizar sus datos y evaluar el neurodesarrollo. El protocolo fue aprobado por el Comité de Ética de la Facultad de Medicina de la Universidad de la República.

\section{Resultados}

En Uruguay, en el período comprendido entre los años 2013 y 2018, se notificaron al MSP 34 casos de HHR, de los cuales 31 cumplían con los criterios de inclusión.
Se analizaron 30, 16 varones y 14 niñas. Uno de ellos se excluyó por imposibilidad de recuperar los datos. La distribución anual de los casos incluidos fue cuatro en 2014 , cuatro en 2015, cuatro en 2016, tres en 2017 y 15 en 2018 (lo que corresponde a 2; 2; 2; 1,6 y 9 casos cada 100.000 dosis de vacuna pentavalente distribuida por año, respectivamente).

La media de edad de aparición del evento fue tres meses, mediana y moda dos meses. El evento ocurrió luego de la primera dosis en 21 niños, luego de la segunda en siete, de la tercera en un caso y de la cuarta en otro.

Las manifestaciones clínicas notificadas fueron: cambio en el tono muscular 28, palidez 24, hipo-reactividad 23 y cianosis 11 . Considerando los niveles de certeza de Brighton, 20 casos se clasificaron en el nivel 1, uno como nivel 2 y ocho como nivel 3. Los síntomas y signos asociados a la tríada diagnóstica se muestran en la Tabla 2.

En la Tabla 3 se muestran los datos de la latencia entre el evento y la vacunación y la duración del evento. La mayoría fue de breve duración y en las primeras horas post vacunación.

La recuperación fue espontánea en 28 de los 30 casos. Se realizaron maniobras de reanimación básica en dos. En la evolución, dos niños recibieron medidas terapéuticas, uno oxigenoterapia y antimicrobianos por diagnóstico presuntivo inicial de infección del sistema nervioso central que se descarta; y otro, luego de maniobras de reanimación básica, adenosina y amiodarona, por taquicardia sinusal mantenida. Este último permaneció dos días en la unidad de cuidados intensivos y se otorgó alta con diagnóstico de taquicardia sinusal de probable mecanismo vasovagal.

Requirieron hospitalización 29 niños: tres en unidad

Tabla 2. Estudio de eventos HHR post-vacuna pentavalente, Uruguay, 2014-2018 (n: 30)*. Síntomas y signos asociados a la tríada diagnóstica

\begin{tabular}{lc}
\hline Síntoma o signo & n de casos \\
\hline Fiebre & 7 \\
Llanto & 5 \\
\hline Supraversión ocular & 4 \\
\hline Apnea & 4 \\
\hline Deposiciones líquidas & 3 \\
Irritabilidad & 3 \\
\hline Vómito & 2 \\
\hline Anisocoria & 1 \\
\hline Taquicardia & 1 \\
Movimientos oculares & 1 \\
\hline Desaturación & 1 \\
Movimientos anormales & 1 \\
\hline Exantema morbiliforme & 2 \\
\hline HHR: hipotonía-hiporrespuesta. & \\
\hline
\end{tabular}


de cuidados intensivos (UCI), 12 en área de observación de emergencia y 12 en cuidados moderados. En dos se desconoce el nivel de hospitalización.

Fue posible determinar la duración de la hospitalización en 24 de los 29 niños hospitalizados. La media de la estadía hospitalaria fue $18 \mathrm{~h}$ (0-3 días).

Se obtuvo el dato de los estudios complementarios realizados en 18 de los 29 hospitalizados: electroencefalograma 12, estudio del líquido cefalorraquídeo tres y ecocardiograma cuatro.

Un niño reiteró el episodio en las primeras $24 \mathrm{~h}$ y completó el esquema de vacunación con pentavalente sin recurrencias.

En 11 se desconoce si presentaron o no recurrencias del evento. Presentaron recurrencias con dosis posteriores de la vacuna pentavalente cinco niños. Uno de ellos presentó además eventos no relacionados a la vacunación y recibió las vacunas correspondientes sin el componente anti pertussis.

Completaron el esquema de vacunación correspondiente a su edad actual 27 de los 30 niños. En tres casos se administraron los componentes de la vacuna pentavalente sin el componente pertussis. Los tres pacientes que fueron hospitalizados en UCI recibieron las dosis posteriores de vacuna pentavalente.

Se realizó el tamizaje del neurodesarrollo en 16 . No se logró coordinar la evaluación en 12 y no dieron su consentimiento para la misma dos. La media de tiempo entre el evento y la evaluación del neurodesarrollo fue 2 años y 2 meses, (rango 9 meses-4 años 10 meses).

En la Tabla 4 se muestra la edad y características de los eventos del grupo de niños evaluados y no evaluados. No se observaron diferencias significativas entre ambos grupos.

Pasaron la prueba de tamizaje 15 de los 16 niños evaluados. En un varón de 20 meses, con contexto sociofamiliar deficitario, se detectó un retraso en el área del lenguaje, con normalidad en las áreas motora, social y de coordinación.

\section{Discusión}

En esta cohorte, la prevalencia, distribución por edad y sexo y las características de los eventos de HHR (dosis de vacuna involucrada, latencia, manifestaciones clínicas, duración) fueron similares a los reportado en publicaciones previas, tanto en países de la región como a nivel internacional ${ }^{7-10}$.

Es probable que la elevada frecuencia de hospitalización y realización de estudios complementarios se relacione con lo inespecífico de las manifestaciones clínicas, especialmente en niños pequeños que pueden corresponder a distintas etiologías (infecciosas, neu-

\begin{tabular}{|c|c|}
\hline Rango (min) & $0-1.440$ \\
\hline Primer cuartil (min) & 120 \\
\hline Mediana (min) & 240 \\
\hline Tercer cuartil (min) & 360 \\
\hline Media (min) & 356 \\
\hline \multicolumn{2}{|c|}{ Duración del evento $(n=21)$} \\
\hline$\leq 5 \min$ & $\mathrm{n}=15$ \\
\hline$>5 a \leq 1 h$ & $n=5$ \\
\hline$>1 \mathrm{~h}$ & $\mathrm{n}=1$ \\
\hline Rango (min) & $0-720$ \\
\hline Media (min) & 44 \\
\hline Primer cuartil (min) & 1 \\
\hline Mediana (min) & 3 \\
\hline Tercer cuartil (min) & 10 \\
\hline
\end{tabular}

Tabla 4. Comparación de algunas de las variables de estudio entre pacientes en los que se evaluó el neurodesarrollo y en los que no

\begin{tabular}{|c|c|c|c|}
\hline Características & $\begin{array}{c}\text { Pacientes } \\
\text { evaluados } \\
(n=16)\end{array}$ & $\begin{array}{c}\text { Pacientes } \\
\text { no evaluados } \\
(n=14)\end{array}$ & \\
\hline Edad (media) (años) & 0,23 & 0,29 & NS \\
\hline Ingreso a UCI (n) & 1 & 2 & NS \\
\hline Mediana de duración evento (min) & $3^{*}$ & $1 * *$ & NS \\
\hline Recuperación espontánea (n) & 16 & 12 & NS \\
\hline Reiteración mismo evento (n) & 1 & 0 & NS \\
\hline Recurrencia con dosis posteriores (n) & 4 & 1 & NS \\
\hline
\end{tabular}

rológicas, cardiovasculares). Si bien la mayoría de los eventos de HHR se presentaron con un nivel de certeza de Brighton 1, el diagnóstico de evento relacionado implica descartar diagnósticos diferenciales, lo que podría justificar la realización de exámenes complementarios y la adopción de diferentes medidas terapéuticas. Realizar el diagnóstico definitivo de HHR post vacunal requiere un análisis sistemático por técnicos con formación en farmacovigilancia. Se trata de un diagnóstico retrospectivo que implica conocer la forma de presentación, recuperación y evolución del evento. Al igual que lo observado en esta cohorte, el evento HHR post vacunal generalmente ocurre en las primeras $48 \mathrm{~h}$ postvacunal, se recupera de forma espontánea y su evolución es benigna ${ }^{6-9}$.

Es de suma importancia, notificar el evento frente a la sospecha al Sistema Nacional de Farmacovigilancia, describiendo con la mayor precisión posible las carac- 
terísticas de éste, basándose sobre todo en la relación temporal con la vacunación.

A pesar que en una proporción importante de niños de esta serie no se pudo determinar la existencia o no de recurrencia con dosis posteriores de vacuna, la proporción encontrada en esta serie fue más alta ( 5 de 19 niños) que lo reportado por la bibliografía. Si bien son muy pocos los estudios en el concierto internacional que evalúan estos aspectos, se comunica que este hecho es poco frecuente, con series que no evidencian recurrencia mayor a $2 \%{ }^{15-17}$. Es probable que factores como los que explican la amplia variación en la ocurrencia puedan también explicar el hallazgo de variaciones en la recurrencia. Entre ellos, las variaciones en la composición de las vacunas, esquemas vacunales diferentes entre países, tipo de vigilancia epidemiológica y definición de caso adoptada ${ }^{18}$. Sería interesante analizar este hecho particular para identificar si estos $u$ otros factores pueden incidir en las variaciones en la frecuencia de la aparición y recurrencia de este síndrome en nuestro país.

El MSP ha realizado recomendaciones frente a la aparición de un ESAVI neurológico grave vinculado al componente pertussis de la vacuna pentavalente o DPT (convulsión, HHR o llanto persistente), recalcando que no constituye una contraindicación absoluta para la vacunación con futuras dosis. Los niños que experimenten estos episodios deben ser observados clínicamente durante al menos $12 \mathrm{~h}$ tras la administración de la siguiente dosis de vacuna. En los casos en que alguno de estos eventos se repita, se debe individualizar la conducta posterior ${ }^{13}$. En este estudio, aquellos en los que se optó por no administrar el componente pertussis de la vacuna pentavalente, no cumplían con una verdadera contraindicación.

Evaluar el desarrollo a largo plazo de los niños que han tenido HHR contribuye a conocer el perfil de seguridad y fortalecer la confianza en las vacunas. Si bien en este estudio el número de niños incluidos es escaso, no se analizaron los aspectos socio-demográficos y se utilizó una prueba de tamizaje, la herramienta utilizada fue validada a nivel nacional para ser utilizada por médicos del primer nivel de atención con una buena sensibilidad. Resulta interesante que no se detectaron alteraciones (retrasos ni desvíos) en área alguna del neurodesarrollo en los niños evaluados. El retraso del lenguaje detectado en uno de los niños requiere profundización diagnóstica y podría estar relacionado con factores ambientales. Se destaca que, al comparar los niños evaluados con los no evaluados, no se observaron diferencias significativas en las principales características de los eventos que podrían influir en la aparición de complicaciones a largo plazo en el neurodesarrollo. Estos hallazgos son coincidentes con lo descrito en la literatura médica sobre lo benigno de los episodios ${ }^{6-9}$.

La importancia y gravedad de este tipo de ESAVI parece estar dada por el impacto que tiene en el momento que ocurre: la necesidad de asistencia de forma urgente, tiempos de observación hospitalaria $\mathrm{y}$, en algunas oportunidades, la necesidad de descartar diagnósticos diferenciales.

Resulta fundamental el hecho de que la mayoría de los niños que tuvieron HHR completaron su esquema vacunal adecuadamente según su edad, dado que el beneficio de estar correctamente vacunados supera ampliamente el riesgo de padecer este tipo de eventos, que como se describe en la literatura médica y parece indicar en esta serie, no implica complicaciones a largo plazo $^{12}$.

\section{Conclusiones}

En los casos estudiados, la HHR se presentó como un evento breve y autolimitado, con características similares a las descritas en la bibliografía, que requirió en la mayor parte de los casos, observación clínica en un centro hospitalario, sin complicaciones graves a corto plazo. A pesar de las limitaciones del estudio, en el tamizaje del neurodesarrollo realizado en promedio a los 2 años del evento, no se encontraron retrasos ni desvíos en los niños evaluados.

\section{Referencias bibliográficas}

1.- Kohl K S, Bonhoeffer J, Miles Braun M, Chen R T, Duclos P, Heijbel H, et al. The Brighton Collaboration: Creating a global standard for case definitions (and guidelines) for adverse events following immunization. Advances in Patient Safety: From Research to Implementation (Volume 2: Concepts and Methodology). Henriksen K, Battles JB, Marks ES, et al., editors. Rockville (MD): Agency for Healthcare Research and Quality (US); 2005 Feb. pp: 87-102. Disponible en https://www.ncbi.nlm.nih.gov/books/ NBK20507/

2.- Rodríguez A, Zunino C, Speranza N, Fernández S, Varela A, Picón T, et al. Seguimiento de eventos supuestamente atribuibles a la vacunación e inmunización (ESAVI) graves notificados al Ministerio de Salud Pública entre 2010 y 2014. Rev Méd Urug. 2017; 33 (1) 47-58. Disponible en: http://www.rmu.org.uy/ revista/2017v1/art6.pdf

3.- Organización Panamericana de la Salud. División de Vacunas e Inmunización. Vacunación Segura: cómo enfrentar los eventos supuestamente atribuidos a la vacunación o inmunización. Washington, D.C.: 2002. Disponible en: http://www.who. int/immunization_safety/publications/aefi/en/ vacunacion_segura_S.pdf [Consulta: 16 de julio de 2019].

4.- Uruguay. Ministerio de Salud Pública. Vacunas. Disponible en: http://www.msp.gub.uy/noticia/ vacunas [Consulta: 16 de julio de 2019].

5.- Ministerio de Salud Pública. Informe del Sistema Notificación de Efectos adversos supuestamente atribuibles a la vacunación o inmunización. Montevideo, 2018 Disponible 


\section{Vacunología}

early childhood: Case definition and guidelines

en: https://www.gub.uy/ministerio-saludpublica/sites/ministerio-salud-publica/files/ documentos/publicaciones/Esavi\%202018. pdf [Consulta: 16 julio 2019]

6.- Galindo B, Concepción D, Galindo M. Episodio de hipotonía e hiporreactividad posterior a la aplicación de vacunas en Cuba durante el período 2012-2013. Rev Cubana Med Trop. 2015; 67 (3). Disponible en: http://scielo.sld. $\mathrm{cu} /$ scielo.phpscript $=$ sci_arttext\&pid=S037507602015000300008\&lng=es

7.- Díaz O, Martínez-Pajares J, Trigo J, RamosDiaz J. Episodio de hipotonía-hipo-rreactividad posvacunación con DTPa. Arch. Argent. Pediatr. 2013; 111 (4): 97-100. Disponible en: https://www.sap.org.ar/docs/publicaciones/ archivosarg/2013/v111n4a16.pdf

8.- Velasco J, Montero D A, Guzmán M. Episodio hipotonía-hiporreactividad posterior a la inmunización con vacuna combinada con pertussis de células enteras. Reporte de un caso. Rev Chil Pediatr. 2017; 88 (6): 771-7. doi: 10.4067/S037041062017000600771.

9.- Buettcher M, Heininger U, Braun M, Bonhoeffer J, Halperin S, Heijbel H, et al. The Brighton Collaboration HHE Working Group. Hypotonic-hyporesponsive episode (HHE) as an adverse event following immunization in for data collection, analysis, and presentation. Vaccine 2007: 25: 5875-81. doi: 10.1016/j. vaccine.2007.04.061.

10.- Baraff L J, Shields W D, Beckwith L, Strome G, Marcy S M, Cherry J D, et al. Infants and children with convulsions and hypotonichyporesponsive episodes following diphtheriatetanus-pertussis immunization: Follow-up Evaluation. Pediatrics 1988. 81 (6); 789-94. PMID: 3259305

11.- Castellano V, Pacchiotti A, Umido V, Lamy P, Nolte M F, Gentile A. Episodios de Hipotonía Hiporrespuesta (EHH) postvacunación: reporte de una serie de casos. Rev. Hosp. Niños (B. Aires) 2018; 60 (268): 29-33. Disponible en: http://revistapediatria.com.ar/wp-content/ uploads/2018/05/268-06-Episodios-dehipotonia.pdf

12.- DuVernoy T S, Braun M. The VAERS Working Group. Hypotonic-hyporesponsive episodes reported to the Vaccine Adverse Event Reporting System (VAERS), 1996-1998. Pediatrics 2000; 106 (4): e52. doi: 10.1542/ peds.106.4.e52

13.- Ministerio de Salud Pública. Guía Nacional de vacunación en situaciones especiales. Montevideo, 2018. Disponible en: https:// www.gub.uy/ministerio-salud-publica/sites/ ministerio-salud-publica/files/documentos/ publicaciones/Vacunacion $\% 20 \mathrm{en} \% 20$ situaciones\%20especiales.pd

14.- Giachetto G, Santoro A, Pérez M, Massa F. Validación de un instrumento para la detección oportuna de problemas de desarrollo en menores de cinco años en Uruguay. Rev Med Urug. 2020; 36 (1): 28-38. doi: https://doi. org/10.29193/RMU.36.1.4

15.- Guetsop J, De Serres G, Kiely M, Gariépy M, Rouleau I, Top K A, et al. Risk of recurrence of adverse events following immunization: a systematic review. Pediatrics 2017; 140 (3):-15. doi: 10.1542/peds.2016-3707.

16.- Zafack J G, Toth E, Landry M, Drolet J P, Top K A, De Serres G. Rate of recurrence of adverse events following immunization: results of 19 years of surveillance in Quebec, Canada. Infect Dis J. 2019; 38 (4): 377-83. doi: 10.1097/ INF.0000000000002162.

17.- Goodwin H, Nash M, Gold M, Heath T C, Burgess M A. Vaccination of children following a previous hypotonic-hyporesponsive episode. J Paediatr Child Health. 1999; 35 (6): 549-52. doi: 10.1046/j.1440-1754.1999.00419.x

18.- Chen R T, Davis R L, Sheedy K M. Safety of Immunizations. In: Plotkin SA, Orenstein WA, editors. Vaccines. 4th ed. Philadelphia: Saunders; 2004. p. 1557-81. 